\title{
Auto-Landing Guidance System Design for Smart UAV
}

\author{
Byoung-Mun Min*, Hyo-Sang Shin* and Min-Jea Tahk** \\ Division of Aerospace Engineering, Department of Mechanical Engineering \\ Korea Advanced Institute of Science and Technology(KAIST), Daejeon, Korea 305-701
}

\author{
Boo Min Kim* and Byoung Soo Kim** \\ School of Mechanical and Aerospace Engineering \\ Gyeongsang National University, Jinju, Korea 660-701
}

\begin{abstract}
This paper deals with auto-landing guidance system design applicable to Smart UAV(Unmanned Aerial Vehicle). The proposed guidance law generates horizontal position, velocity and altitude commands in the longitudinal channel and heading angle command in the lateral channel to track a predetermined trajectory for automatic landing. The longitudinal guidance commands are derived from an approximated dynamic equations in vertical plane. These longitudinal guidance commands are appropriately distributed to each control input as the flight mode of Smart UAV is changed. The concept of VOR(VHF Omni-directional Range) guidance system is applied to generate the required heading angle commands to eliminate the lateral deviation from the desired trajectory. The performance of the proposed guidance system for Smart UAV is evaluated using the nonlinear simulation. Simulation results show that the proposed guidance system for auto- landing provides good tracking performance along the predetermined landing trajectory.
\end{abstract}

Key Word : Auto-landing, Guidance system, Smart UAV, Flight mode

\section{Introduction}

Tilt-rotor aircraft hold the hover performance and control of a helicopter as well as the cruise speed and efficiency of turbo-prop airplane. This type aircraft shows various dynamic characteristics as the flight mode is changed. There are three main flight modes, i.e. helicopter mode, airplane mode, and transition mode in the tilt-rotor aircraft. Because of these different flight modes, the control problem of tilt-rotor aircraft is very difficult compared to that of the conventional airplane. In the airplane mode, conventional control surfaces, such as elevator, aileron, and rudder, provide a primary control effect. Whereas, in the helicopter mode, control is conducted by collective and cyclic pitches of the prop-rotors mounted on wing tip.

The flight control design using classical approach can be performed in such a way of yielding linear models at several reference flight conditions, designing linear controller for each condition, and blending these point designs with gain scheduling scheme. To date, since the classical approach has been successfully applied to various systems and historically proven the reliability, it has been mainly used for aircraft flight control design. However, control system

\footnotetext{
* Ph. D. Student

** Professor

E-mail : mjtahk@fdcl.kaist.ac.kr Tel : 042-869-3718 Fax : 042-869-3710

*** Associate Professor
} 
design of tilt-rotor aircraft by classical technique is tedious and time-consuming process because of its highly nonlinear and uncertain dynamics. Also, the required performances might not be satisfied in the whole flight envelope. To overcome these problems, various nonlinear control techniques have been studied and implemented in the flight control system. The most widely studied approach is adaptive nonlinear control technique using feedback linearization[1,2]. The key idea of feedback linearization is to map a nonlinear systems to a linear system through a coordinate transformation. However, this approach is quite limited in that it relies on exact knowledge of the plant dynamics. In recent year, adaptive nonlinear control combined with artificial neural network has been developed and applied to the flight control system of various aircraft models. The main role of neural network is to compensate an imperfect dynamic inversion model that is generated at a single nominal operating point [3-5]. Application of adaptive control technique using neural network to tilt-rotor aircraft is precisely described in Ref. [6]. In Ref. [7] and [8], neural networks are used to control super-maneuverable aircraft.

The main objective of this paper is to design the guidance system applicable to automatic landing problem of tilt-rotor type Smart UAV. To perfectly achieve this purpose, the inner-loop SCAS(Stability and Control Augmentation System) is designed by using the neural network based on adaptive control scheme and DMI(Dynamic Model Inversion). In this paper, dynamic model inversion is performed at a single operating flight condition at transition mode of Smart UAV. Longitudinal guidance commands are derived from a simplified force equations in vertical plane. The concept of VOR(VHF Omni-directional Range) guidance system[9,10] is adopted for lateral channel. The outer-loop control system with linear controller is designed to integrate and implement the proposed guidance law. All controller gains are selected by CEALM (Co-Evolutionary Augmented Lagrangian Method)[11,12]. The performance of the proposed guidance system is evaluated according to auto-landing scenario.

\section{Inner-loop SCAS Design}

In this section, we will briefly introduce the inner-loop SCAS design procedure of Smart UAV. Adaptive neural network control technique based on DMI was used to design the inner-loop SCAS. The designed inner-loop SCAS is an augmentation system of ACAH(Attitude Command Attitude Hold) type. In this augmentation system, the attitude control of aircraft is performed by means of constituting control inputs from the attitude commands.

\section{Dynamic Model Inversion}

Generally, a nonlinear system of $n$ degrees of freedom can be expressed as

$$
\ddot{x}=f(x, \dot{x}, \delta)
$$

where $x(t) \in R^{n}$ and $\dot{x}(t) \in R^{n}$ are the state variables and $\delta(t) \in R^{m}$ is the control variable. The function $f(\cdot)$ is a mapping from a domain $R^{n} \times R^{n} \times R^{m}$ into $R^{n}$. If $n=m$, it is called a square system. If $f(x, \dot{x}, \cdot)$ is invertible and all states are measurable, Eq. (1) can be transformed to the form

$$
\begin{gathered}
\ddot{x}=u \\
u=f(x, \dot{x}, \delta)
\end{gathered}
$$

where $u(t) \in R^{n}$ is the pseudo-control variable. The inverse transformation of the second line in Eq. (2) is

$$
\delta=f^{-1}(x, \dot{x}, u)
$$

If the function $f$ is perfectly known, one may obtain the exact linearized system as in Eq. (2). 
However, the information of $f$ always contains some errors caused by the model uncertainty and assumption in mathematical modeling.

Considering the inversion error, the first equation in Eq. (2) is expressed as

$$
\ddot{x}=u+\Delta^{\prime}(x, \dot{x}, u)
$$

In this equation, $\Delta^{\prime}: R^{n} \times R^{n} \times R^{m} \rightarrow R^{n}$ is a mapping representation of the inversion error. It is a function of the state variable and the pseudo-control input

$$
\Delta^{\prime}(x, \dot{x}, u)=f(x, \dot{x}, \hat{\delta})-f(x, \dot{x}, \delta)
$$

where $\widehat{\delta}$ represents approximate inversion of the control input due to the model uncertainties.

\section{Adaptive Control Using Neural Network}

In general, PID-type controller is used to satisfy the flying quality of the aircraft stability and performance in the feedback linearization. In this section, we describe how to design the PD-type controller. If the uncertainty of flight dynamics is neglected in the design of PD-type controller, the stability and performance of the control system might be degraded. In the worst case, the closed-loop system might be unstable. In order to avoid this problem, adaptive control technique using neural network is considered in this paper. Now, the adaptive control structure for each channel is chosen as

$$
u_{i}(t)=u_{p d_{i}}(t)+\ddot{x}_{c_{i}}(t)-\hat{u}_{a d_{i}}(t), \quad i=1,2, \cdots, n
$$

where $u_{p d_{i}}$ and $\hat{u}_{p d_{i}}$ represent a PD control and an adaptive control, respectively. The error dynamics can be obtained from Eq. (6) and Eq. (4)

$$
\ddot{\tilde{x}}_{i}+K_{d_{i}} \dot{\tilde{x}}_{i}+K_{p_{i}} \tilde{x}_{i}=\hat{u}_{a d_{i}}-\Delta_{i}^{\prime}(x, \dot{x}, u), \quad i=1,2, \cdots, n
$$

where $\tilde{x}_{i}=x_{c_{i}}-x_{i}$. In Eq. (7), the adaptive term $\hat{u}_{p d_{i}}$ is used to compensate for the inversion error $\Delta_{i}^{\prime}$, so that the steady state error of $\tilde{x}_{i}(t)$ becomes zero. It will be realized by a neural network. In this paper, the adaptive control signal of neural network is chosen as

$$
\hat{u}_{a d_{i}}(t)=\sum_{j=1}^{N} \hat{w}_{i j}(t) \beta_{i j}^{\prime}(x, \dot{x}, u)=\hat{w}_{i}^{T}(t) \beta_{i}^{\prime}(x, \dot{x}, u)
$$

In Eq. (8), $\hat{w}_{i}$ indicates the vector of the weight $\hat{w}_{i j}(t)$ that is updated online, and $\beta_{i j}(\cdot)$ is a basis function of the neural network. In this paper, the weight update rule is selected that the error dynamics expressed in Eq. (7) is bounded in the sense of Lyapunov stability[13].

$$
\dot{\hat{w}}_{i}(t)=\left\{\begin{array}{ll}
-\gamma_{i} e_{i}^{T} P_{i} b \beta_{i}^{\prime}(x, \dot{x}, u) & \text { for }\left\|e_{i}\right\|_{P_{i}}>E_{i} \\
0 & \text { for }\left\|e_{i}\right\|_{P_{i}} \leq E_{i}
\end{array}, \quad i=1,2, \cdots, n\right.
$$

where $\left\|e_{i}\right\|_{P_{i}}=\sqrt{e_{i}^{T} P_{i} e_{i}}$. The positive constant $\gamma_{i}$ is the learning rate and $P_{i}$ is a symmetric, positive definite matrix given as

$$
P_{i}=\left[\begin{array}{cc}
\frac{K_{d_{i}}}{2 K_{p_{i}}}+\frac{K_{p_{i}}}{2 K_{d_{i}}}\left(1+\frac{1}{K_{p_{t}}}\right) & \frac{1}{2 K_{p_{i}}} \\
\frac{1}{2 K_{p_{i}}} & \frac{1}{2 K_{d_{i}}}\left(1+\frac{1}{K_{p_{t}}}\right)
\end{array}\right]
$$




\section{Inner-loop SCAS Design of Smart UAV}

The control architecture of ACAH logic for Smart UAV is shown in Fig. 1. The linearized rotational dynamics of Smart UAV at an arbitrary trim flight condition can be written as

$$
\left[\begin{array}{c}
\dot{p} \\
\dot{q} \\
\dot{r}
\end{array}\right]=\hat{A}\left[\begin{array}{lllllll}
\Delta u & \Delta w & q & \Delta v & p & r & \Delta \delta_{C O L}
\end{array}\right]^{T}+\hat{B}\left[\begin{array}{c}
\Delta \delta_{L A T} \\
\Delta \delta_{L O N} \\
\Delta \delta_{P E D}
\end{array}\right]
$$

Matrices $A$ and $\widehat{B}$ are composed of moment of inertia, stability and control derivatives. In this paper, the linearized model in Eq. (11) is obtained at the transition mode with velocity of 120 knots and nacelle angle of $60 \mathrm{deg}$. From Eq. (11), the control inputs are determined as

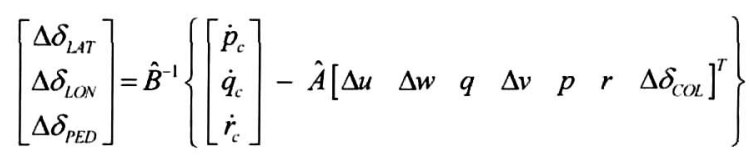

The PD controllers are designed in each control channel as follows:

$$
\begin{aligned}
& u_{\phi}=K_{\phi}^{p}\left(\phi_{c}-\phi\right)+K_{\phi}^{d}\left(\dot{\phi}_{c}-\dot{\phi}\right) \\
& u_{\theta}=K_{\theta}^{p}\left(\theta_{c}-\theta\right)+K_{\theta}^{d}\left(\dot{\theta}_{c}-\dot{\theta}\right) \\
& u_{\psi}=K_{\psi}^{p}\left(\psi_{c}-\psi\right)+K_{\psi}^{d}\left(\dot{\psi}_{c}-\dot{\psi}\right)
\end{aligned}
$$

where $u_{\psi}=\dot{\phi}, u_{\theta}=\dot{\theta}$, and $u_{\psi}=\ddot{\psi}$ denote pseudo-control variables. Proportional gain $K_{i}^{p}$ and derivative gain $K_{i}^{d}$ are selected to satisfy ADS-33D Handling Quality[14]. The required performances of attitude control system and determined control gains are summarized in Table 1.

Table 1. Requirement for attitude control system and PD gains

\begin{tabular}{|r|c|c|}
\hline Performance requirement & Natural freq. / Damping ratio & PD gains \\
\hline Settling time : $\quad t_{s}=0.8 \mathrm{sec}$ & $\omega_{n}=6.0 \mathrm{rad} / \mathrm{sec}$ & $K_{i}^{p}=36.0$ \\
\hline Overshoot : $\quad M_{p}=0 \%$ & $\zeta=1.0$ & $K_{i}^{d}=12.0$ \\
\hline
\end{tabular}

Since the dynamic inversion is performed at an arbitrary single flight condition, the approximated linear dynamics of Eq. (11) is not perfect. Moreover, the model uncertainties might degrade the performance of dynamic inversion. Because of this reason, adaptive signals are augmented to compensate for dynamic inversion error.

$$
u_{i}(t)=u_{p d_{i}}(t)+\ddot{x}_{c_{i}}(t)-\hat{u}_{a d_{i}}(t), \quad i=\phi, \theta, \psi
$$

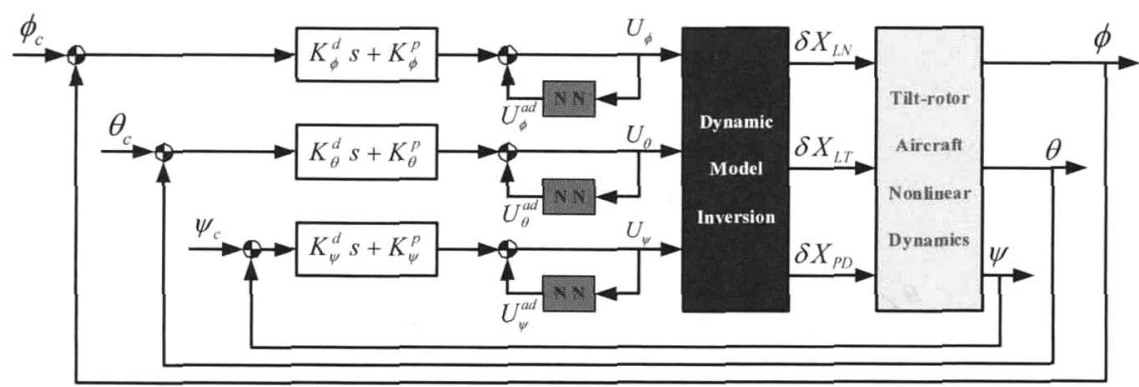

Fig. 1. The control architecture of ACAH logic for Smart UAV 
At this time, the adaptive control signal of neural network is chosen as

$$
\hat{u}_{a d,}(t)=\sum_{j=1}^{N} \hat{w}_{i j}(t) \beta_{i j}^{\prime}(x, \dot{x}, u), \quad i=\phi, \theta, \psi
$$

In this paper, Sigma-Phi Neural Network (SPNN) is used to adaptation, and the basis functions in each control channel are chosen as follows:

$$
\begin{aligned}
& \hat{u}_{a d_{\phi}}=\sum_{i=1}^{N} \hat{w}_{\phi j} \beta_{\phi j}\left(U_{\delta}, W_{\delta}, V p_{\delta}, \phi_{\delta}, p_{\delta}, u_{\phi}\right) \\
& \hat{u}_{a d_{\theta}}=\sum_{i=1}^{N} \hat{w}_{\theta j} \beta_{\theta j}\left(U_{\delta}, W_{\delta}, V p_{\delta}, \theta_{\delta}, p_{\delta}, u_{\theta}\right) \\
& \hat{u}_{a d_{v}}=\sum_{i=1}^{N} \hat{w}_{\psi j} \beta_{\psi j}\left(U_{\delta}, W_{\delta}, V p_{\delta}, \psi_{\delta}, p_{\delta}, u_{\psi}\right)
\end{aligned}
$$

\section{Guidance System Design}

As the nacelle angle varies from $0 \mathrm{deg}$. to $90 \mathrm{deg}$. during landing procedure, Smart UAV shows different dynamic characteristics. Consequently, derivation of guidance law and design of outer-loop guidance system is very difficult problem. In this paper, the guidance law applicable to auto-landing problem of Smart UAV is proposed. Guidance commands in longitudinal direction are generated to track the horizontal position, velocity, and altitude of the predetermined reference trajectory. Lateral guidance command is generated to eliminate the lateral deviation error between the reference trajectory and the actual trajectory. In this section, we will describe the outer-loop guidance system design of Smart UAV.

\section{Longitudinal Guidance Law}

From Fig. 2, the simplified force equation of Smart UAV in horizontal and vertical direction can be expressed as

$$
\begin{aligned}
& m \dot{v}_{x}=T \sin \left(\beta_{M}-\theta\right)+F_{x} \\
& m \dot{v}_{h}=T \cos \left(\beta_{M}-\theta\right)-m g+F_{h}
\end{aligned}
$$

where $T$ is a thrust produced by rotor, $F_{x}$ and $F_{h}$ are aerodynamic forces in horizontal and vertical direction, and $\beta_{M}$ is a nacelle angle that is $90 \mathrm{deg}$. in the airplane mode and $0 \mathrm{deg}$. in the helicopter hovering mode. If the velocity and nacelle angle are given, then Eq. (17) can be linearized as

$$
\begin{aligned}
& m \dot{v}_{x}=-T^{*} \cos \beta_{M} \delta \theta+\sin \beta_{M} \delta T+\delta F_{x} \\
& m \dot{v}_{h}=T^{*} \sin \beta_{M} \delta \theta+\cos \beta_{M} \delta T+\delta F_{h}
\end{aligned}
$$

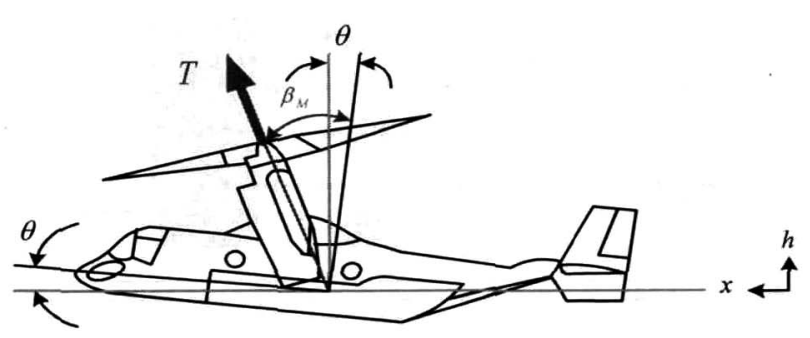

Fig. 2. Thrust geometry in vertical plane 
where superscript $*$ and $\delta$-term indicate trim value and small perturbation, respectively. Because $\delta F_{h}$ has the same sign of $\delta \Theta, \delta F_{h}$ can be roughly approximated as $\delta F_{h}=c \delta \theta(c>0)$, and $\delta F_{x}$ is negligible in the case that $\delta \Theta$ is small. Also, $\delta T$ can be modeled as $\delta T=k \delta \xi$, where $\xi$ denotes the collective pitch angle of rotor. Using these relationships, Eq. (18) can be rewritten as

$$
\begin{aligned}
& m \dot{v}_{x}=-T^{*} \cos \beta_{M} \delta \theta+k \sin \beta_{M} \delta \xi \\
& m \dot{v}_{h}=\left(T^{*} \sin \beta_{M}+c\right) \delta \theta+k \cos \beta_{M} \delta \xi
\end{aligned}
$$

From Eq. (19), control inputs $\delta \Theta$ and $\delta \xi$ are determined as

$$
\left[\begin{array}{c}
\delta \xi \\
\delta \theta
\end{array}\right]=\left[\begin{array}{cc}
\frac{m T^{*}}{k T_{0}} \cos \beta_{M} & \frac{m T^{*}}{k T_{0}} \sin \beta_{M}+\frac{m c}{k T_{0}} \\
\frac{m}{T_{0}} \sin \beta_{M} & -\frac{m}{T_{0}} \cos \beta_{M}
\end{array}\right]\left[\begin{array}{l}
\dot{v}_{x} \\
\dot{v}_{h}
\end{array}\right]
$$

where $T_{0}=T^{*}+c \sin \beta_{M}$. As the nacelle angle approaches to 0 deg., i.e. helicopter mode, Eq. (20) can be simplified

$$
\left[\begin{array}{c}
\delta \xi \\
\delta \theta
\end{array}\right]=\left[\begin{array}{ll}
\frac{m}{k} \cos \beta_{M} & \frac{m}{k} \sin \beta_{M} \\
\frac{m}{T^{*}} \sin \beta_{M} & -\frac{m}{T^{*}} \cos \beta_{M}
\end{array}\right]\left[\begin{array}{l}
\dot{v}_{x} \\
\dot{v}_{h}
\end{array}\right]
$$

\section{Lateral Guidance Law}

For the typical fixed-wing aircraft, radio navigation system are very commonly used and the VOR guidance system shown in Fig. 3 is one of the most effective and popular method to achieve automatic tracking of lateral path during landing procedure. In this paper, this concept is adopted to lateral guidance system. In the VOR system, the output signal from the VOR receiver equipped in the aircraft is proportional to the error angle $\Gamma$. This signal is used to calculate the lateral deviation distance of the aircraft from the center-line of the VOR beam. The role of outer-loop guidance system in lateral direction reduces the error angle $\Gamma$ for the purpose of eliminating the lateral deviation distance $d$. From Fig. 3, the lateral deviation distance is determined as

$$
\dot{d}=V_{T} \sin \left(\psi-\psi_{\text {ref }}\right), \quad d=\int V_{T} \sin \left(\psi-\psi_{\text {ref }}\right)
$$

\section{Outer-loop Guidance System Design}

In the airplane mode, forward direction control and vertical direction control are realized by collective input $X_{C O L}$ and longitudinal cyclic input $X_{L O N}$, respectively. In the helicopter mode, the

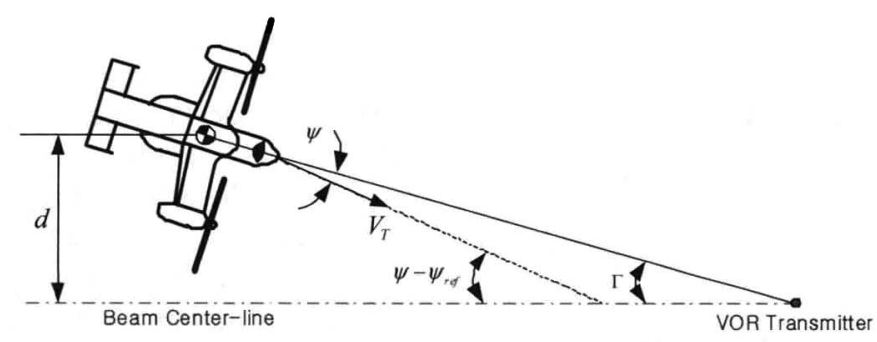

Fig. 3. Geometry of VOR guidance system 


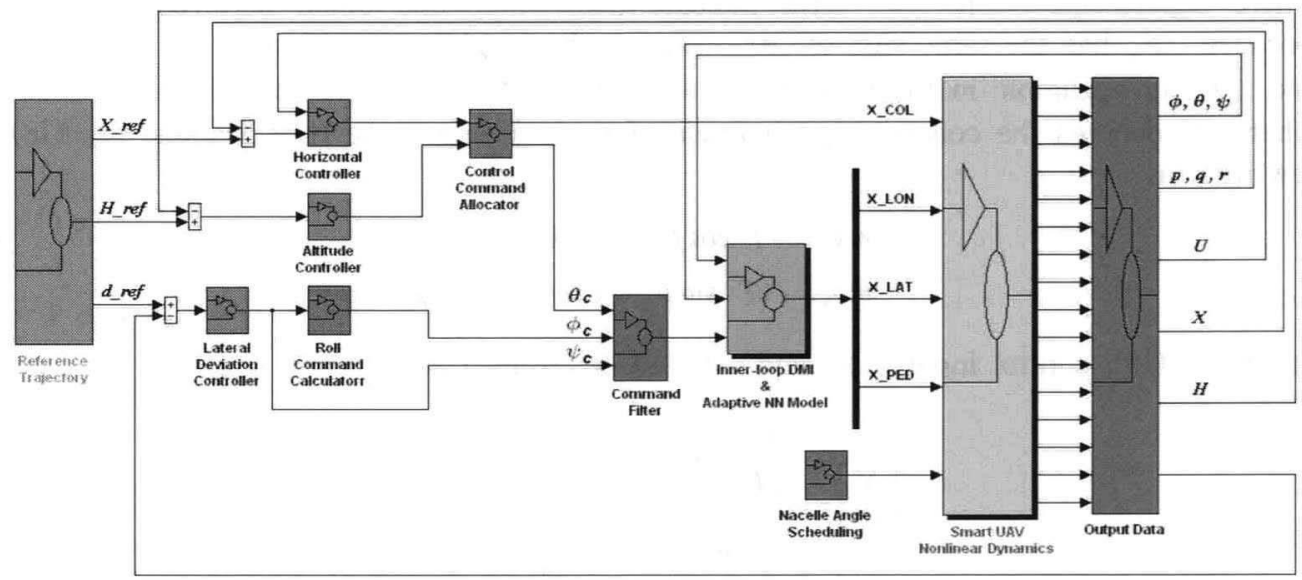

Fig. 4. Guidance system for auto-landing of Smart UAV

roles of control are exchanged. Fig. 4 shows the guidance system of Smart UAV. The outer-loop guidance system is composed of position $G_{x}(s)$ and velocity controller $G_{u}(s)$ for forward direction control, and altitude controller $G_{h}(s)$ for vertical direction control. In the lateral direction, lateral deviation controller $G_{d}(s)$ produces the heading angle command to eliminate the deviation error and roll angle command is determined for coordinated level tern. The gains of these controllers are determined by using CEALM algorithm[11] and are summarized in Table 2.

Table 2. Requirement for attitude control system and PD gains

\begin{tabular}{|c|c|c|c|c|}
\hline & $G_{u}(s)$ & $G_{x}(s)$ & $G_{h}(s)$ & $G_{d}(s)$ \\
\hline Proportional gain & $K_{p}^{u}=7.38$ & $K_{p}^{x}=22.51$ & $K_{p}^{h}=0.67$ & $K_{p}^{d}=6.0$ \\
\hline Integral gain & $K_{i}^{u}=8.06$ & $K_{i}^{x}=0.003$ & $K_{i}^{h}=0.06$ & $K_{i}^{d}=4.0$ \\
\hline
\end{tabular}

\section{Nonlinear Simulation}

\section{Reference Trajectory Determination for Auto-landing of Smart UAV}

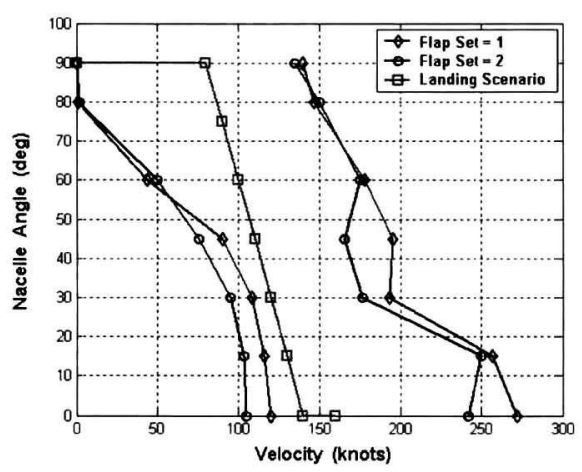

Fig. 5. Conversion corridor

Reference trajectory for auto-landing of Smart $\mathrm{UAV}$ is decided by the information of conversion corridor which is determined by trim flight analysis. Fig. 5 shows the conversion corridor of Smart UAV according to the velocity and the variation of nacelle angle. Where "Flap Set $=1$ " and "Flap Set $=2$ " mean that the flap deflection angles are $0 \mathrm{deg}$. and $45 \mathrm{deg}$., respectively. Based on the conversion corridor, reference trajectory and nacelle angle scheduling scenario for auto-landing is determined in accordance with velocity. Fig. 6 illustrates the shape of predetermined reference trajectory of Smart UAV for auto-landing scenario. In this landing scenario, velocity is decreased to 140 knots maintaining constant altitude of $1000 \mathrm{ft}$ in the airplane mode. At the end of the airplane mode, 


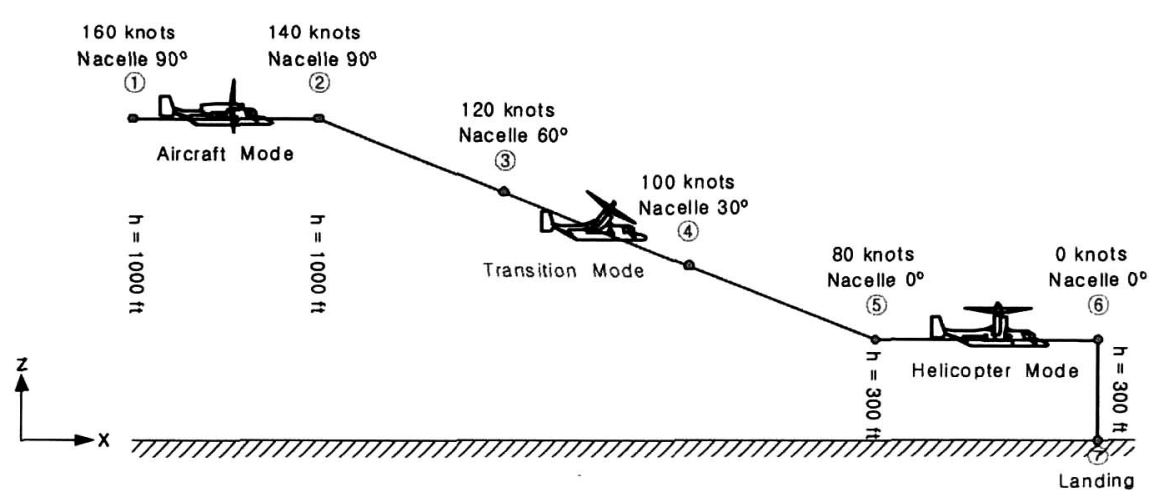

Fig. 6. Predetermined auto-landing scenario of Smart UAV

Smart UAV enters the transition mode. In the transition mode, nacelle angle is tiled gradually to reach 0 deg. with velocity of 80 knots and altitude of $300 \mathrm{ft}$. During transition flight, horizontal distance and altitude of the reference trajectory are determined by considering velocity and flight path angle $\gamma$ of vehicle. After the complete transition flight, Smart UAV flies in the state of forward straight level flight decreasing velocity to 0 knots holds altitude of $300 \mathrm{ft}$. Finally, it decreases altitude to reach $O \mathrm{ft}$ for land in the helicopter hovering mode.

\section{Simulation Results}

Complete nonlinear simulation model of Smart UAV is shown in Fig. 4. This simulation model is programmed using Matlab/Simulink based on the fully nonlinear 6-DOF dynamics. Four control stick models, i.e. collective, longitudinal cyclic, lateral cyclic, and pedal, are included in "Smart UAV Nonlinear Dynamics" block only with saturation limit. These saturation values are limited as $0 \sim 12$ inch in collective input, $-4.8 \sim 4.8$ inch in longitudinal and lateral cyclic input, and $-2.5 \sim 2.5$ inch in pedal input. In this simulation model, dynamic model inversion for inner-loop SCAS is performed as a single operation flight condition in the transition mode with velocity of 120 knots and nacelle angle of $60 \mathrm{deg}$. As a result of dynamic model inversion performed at this condition, an inversion error is occurred at any other flight conditions or flight modes. Outer-loop guidance system is implemented and integrated with inner-loop SCAS. In "Reference Trajectory" block, the predetermined auto-landing trajectory as depicted in Fig. 6 is generated and provided to outer-loop guidance system.

Initially, Smart UAV is in the straight level flight at velocity of 160 knots and altitude of $1000 \mathrm{ft}$ with lateral deviation error of $100 \mathrm{ft}$ from the reference trajectory. Fig. 7 and Fig. 8 show

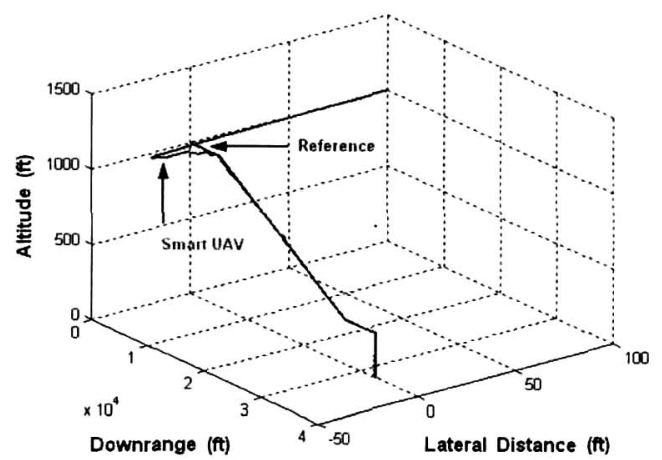

Fig. 7. Three-dimensional flight trajectories

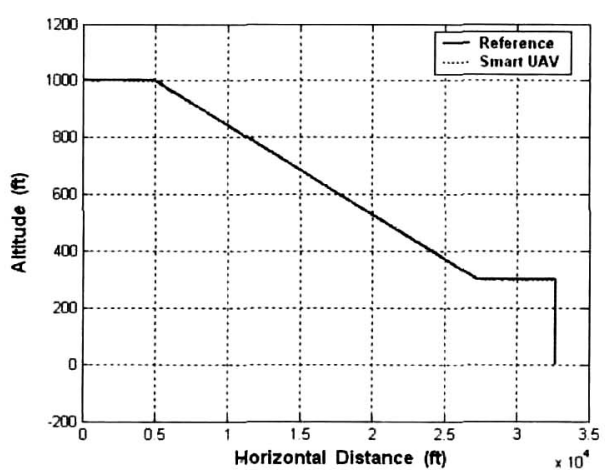

Fig. 8. Flight trajectories in vertical plane 


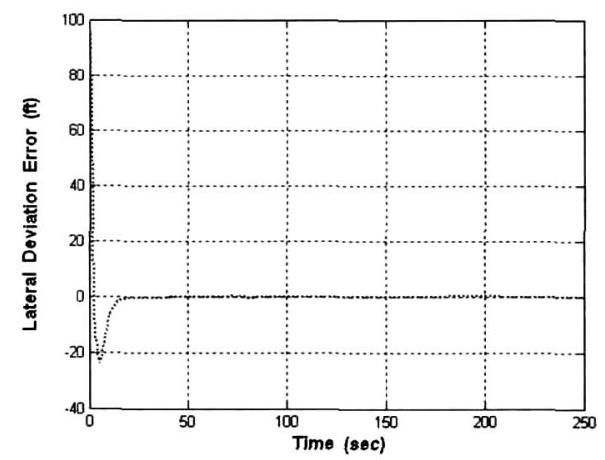

Fig. 9. Time history of lateral deviation error

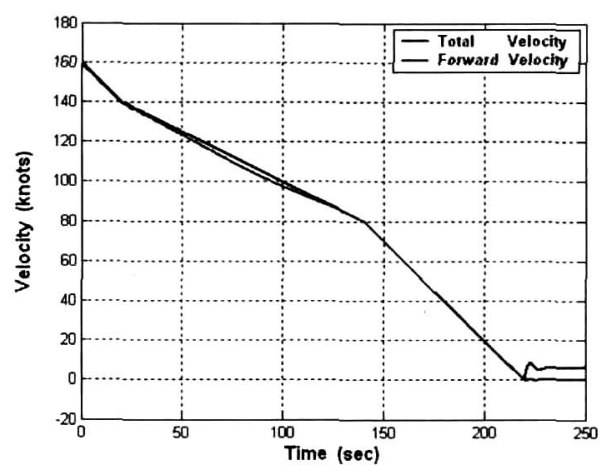

Fig. 11. Time histories of velocities
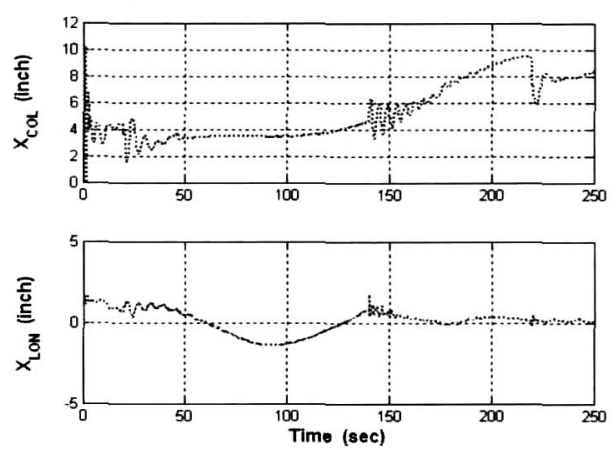

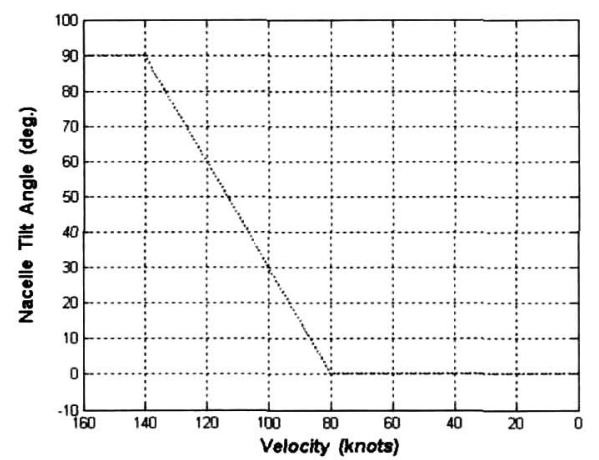

Fig. 10. Variation of nacelle tilt angle
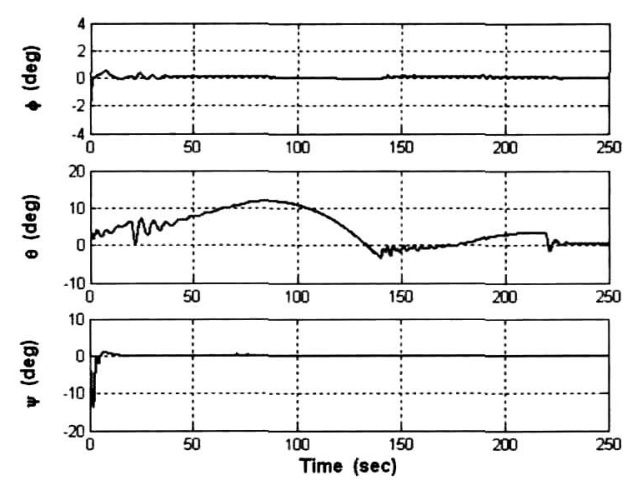

Fig. 12. Time histories of attitude angles
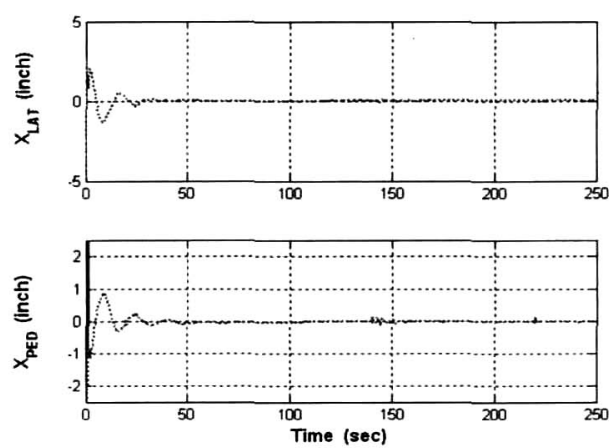

Fig. 13. Time histories of four control stick inputs

three dimensional flight trajectory and its projected trajectory in vertical plane. As can be seen from Figs. 7 and 8, one may easily note that horizontal position and altitude are very tightly controlled by the proposed guidance system. The time history of lateral deviation error is shown in Fig. 9. The lateral deviation error is perfectly eliminated in the airplane mode. Since the controller gain of lateral deviation controller $G_{d}(s)$ is not optimized, the overshoot of lateral deviation error is occurred. Nacelle tilt angle variation with respect to velocity is depicted in Fig. 10. Nacelle tilt angle, which is $90 \mathrm{deg}$. in the airplane mode and $0 \mathrm{deg}$. in the helicopter hovering mode, is reversely defined that of nacelle angle. Nacelle tilt angle command is determined by a 
prescribed scheduling scenario according to the velocity. Time histories of velocities and attitude angles are shown in Fig. 11 and Fig. 12. Also, the variation of four control stick inputs are illustrated in Fig. 13. The oscillatory responses of attitude angles and control stick inputs at the point of flight mode change are observed. It is caused by an abruptly changed longitudinal guidance commands. As previously mentioned, the roles of longitudinal controls are exchanged in the tilt-rotor type aircraft. Generally, forward direction control is realized by collective input and vertical direction control is realized by longitudinal cyclic input in the airplane mode, whereas the realization of control conversely trades in the helicopter mode.

\section{Conclusions}

In this paper, a guidance system of Smart UAV applicable to auto-landing problem has been proposed. The proposed guidance law is derived from a simplified force equations of longitudinal motion. In lateral direction, the concept of VOR system, which is widely adopted to the guidance system of typical fixed-wing aircraft during auto-landing, is directly applied. The inner-loop SCAS is designed using adaptive neural networks based on dynamic model inversion. The designed inner-loop SCAS is ACAH type augmentation system. Simulation results show that the neural network successfully compensates the inversion error caused by dynamic inversion with a single operating condition. The outer-loop control system is designed to implement the proposed guidance law and integrated with the inner-loop SCAS. The proposed guidance system is evaluated by nonlinear simulation with the predetermined reference trajectory for landing. Results from the nonlinear simulation demonstrate precise tracking performance and the applicability of the new guidance system for auto-landing of the tilt-rotor type aircraft.

\section{Acknowledgement}

This paper was performed for Smart UAV Development Program, one of the 21st Century Frontier R\&D Programs funded by the Ministry of Commerce, Industry and Energy of Korea.

\section{References}

1. Baker, R. C., and Charlie, B., 1989, "Nonlinear Unstable Systems", International Journal of Control, Vol. 24, No. 4, pp. 123-145.

2. Hong, K. S., and Kim, C. S., 1993, "Linear Stable Systems", IEEE Transaction on Automatic Control, Vol. 33, No. 11, pp. 1234-1245.

3. Rysdyk, R. T., and Calise, A. J., 1997, "Nonlinear Adaptive Control of Tiltrotor Aircraft sing Neural Networks", SAE/AIAA World Aviation Congress 1997 Proceedings, Anaheim, CA

4. Kim, B. S., and Calise, A. J., 1997, "Nonlinear Flight Control Using Neural Networks", Journal of Guidance, Control, and Dynamics, Vol. 20, No. 1, pp. 26-33.

5. Leitner, J., Calise, A. J., and Prasad, J. V. R., 1997, "Analysis of Adaptive Neural Networks for Helicopter Flight Control", Journal of Guidance, Control, and Dynamics, Vol. 20, No. 5, pp. 972-979.

6. Calise, A. J., and Rysdyk. R. T., 1998, "Nonlinear Adaptive Flight Control Using Neural Networks", IEEE Control Systems, pp. 14-25.

7. Baker, W. L., and Farrell, J. A., 1991, "Learning Augmented Flight Control for High Performance Aircraft", Proceedings of the AIAA Guidance, Navigation, and Control Conference.

8. Steck, J. E., and Rokhasz, K., 1992, "Use of Neural Networks in Control of High Alpha Maneuvers", 30th AIAA Aerospace Sciences Meeting and Exhibit, Reno, NV.

9. Stevense, B. L., and Lewis, F. L., 1992, Aircraft Control and Simulation, John Wiley \& Sons, Inc., New York. 

London.

10. McLean, D., 1991, Automatic Flight Control Systems, Prentice Hall International Ltd.,

11. Tahk, M. J., and Sun, B. C., 2000, "Coevolutionary Augmented Lagrangian Methods for Constrained Optimization", IEEE Transactions on Evolutionary Computation, Vol. 4, No. 2, pp. 114-124.

12. Park, C. S., and Tahk, M. J., 1998, "A Coevolutionary Minimax Solver and Its Application to Autopilot Design", AIAA Guidance, Navigation, and Control Conference, Boston, MA.

13. Khalil, H. K., 2002, Nonlinear Systems, 3rd. ed., Prentice Hall, Inc., Upper Saddle River, New Jersey.

14. Aeronautical Design Standard, 1994, "Handling Qualities Requirements for Military Rotorcraft", $A D S-33 D$, U.S. Army, St. Louis, MO. 